\title{
Front Matter: Volume 10277
}

, "Front Matter: Volume 10277," Proc. SPIE 10277, Adaptive Computing: Mathematics, Electronics, and Optics: A Critical Review, 1027701 (1 March 1994); doi: 10.1117/12.2284872

SPIE Event: SPIE's International Symposium on Optical Engineering and Photonics in Aerospace Sensing, 1994, Orlando, FL, United States 


\section{Contents}

vii Conference Committee

\section{OVERVIEW}

3 Prolegomena to the field

S.-S. Chen, Univ. of North Carolina/Charlotte; H.-J. Caulfield, Alabama A\&M

Univ.

$8 \quad$ Stochastic search

G. L. Bilbro, North Carolina State Univ.

34 Complex and chaotic systems

S.-S. Chen, Univ. of North Carolina/Charlotte

\section{NEURAL NETWORKS}

$47 \quad$ Pulse-coupled neural networks

J. L. Johnson, U.S. Army Missile Command

77 Complexity and chaotic dynamics in a spiking neuron embodiment

N. H. Farhat, S.-Y. Lin, M. H. Eldefrawy, Univ. of Pennsylvania

89

Digital electronic neural networks

D. Hammerstrom, Adaptive Solutions, Inc.

\section{NEW COMPUTER APPROACH}

111 Quantum mechanical computation

J. D. Brasher, Teledyne Brown Engineering

141 Optical neural networks

D. Psaltis, M. Levene, California Institute of Technology

150 Genetic and evolutionary systems

R. E. Smith, Univ. of Alabama

\section{FUZZY SYSTEMS}

177 Optical selectionist approach to optical connectionist systems

H. J. Caulfield, Alabama A\&M Univ.

204 Pattern recognition, neural networks, fuzzy control: what's next?

J. C. Bezdek, Univ. of West Florida

234 Fuzziness in quantum mechanics

A. T. Granik, U dapdifyeheoflapuifirg: Mathematics, Electronics, and Optics: A Critical Review, edited by Su-Shing Chen,

H. John Caulfield, Proc. of SPIE Vol. 10277 (Vol. CR55), 1027701 - ( ) (1994) 2017 SPIE

CCC code: $0277-786 X / 17 / \$ 18 \cdot$ doi: $10.1117 / 12.2284872$

v

Proc. of SPIE Vol. 10277 1027701-1

Downloaded From: https://www.spiedigitallibrary.org/conference-proceedings-of-spie on 25 Apr 2023 Terms of Use: https://www.spiedigitallibrary.org/terms-of-use 
Proc. of SPIE Vol. 10277 1027701-2

Downloaded From: https://www.spiedigitallibrary.org/conference-proceedings-of-spie on 25 Apr 2023 Terms of Use: https://www.spiedigitallibrary.org/terms-of-use 


\title{
Conference Committee
}

\author{
Conference Chairs
}

Su-Shing Chen, University of North Carolina/Charlotte

H. John Caulfield, Alabama A\&M University

Session Chairs

1 Overview

Su-Shing Chen, University of North Carolina/Charlotte

H. John Caulfield, Alabama A\&M University

2 Neural Networks

Demetri Psaltis, California Institute of Technology

Alex T. Granik, University of the Pacific

3 New Computer Approach

Nabil H. Farhat, University of Pennsylvania

Griff L. Bilbro, North Carolina State University

$4 \quad$ Fuzzy Systems

John L. Johnson, U.S. Army Missile Command

James D. Brasher, Teledyne Brown Engineering 